\title{
Trapping Reactions with Randomly Moving Traps: Exact Asymptotic Results for Compact Exploration.
}

\author{
G.Oshanin $^{1}$, O.Bénichou ${ }^{2}$, M.Coppey ${ }^{1}$, and M.Moreau ${ }^{1}$ \\ ${ }^{1}$ Laboratoire de Physique Théorique des Liquides (CNRS - UMR 7600), Université Pierre et Marie Curie, 4 place Jussieu, \\ 75252 Paris Cedex 05, France \\ 2 Laboratoire de Physique de la Matière Condensée, Collège de France, 11 place M.Berthelot, 75231 Paris Cedex 05, France
}

(Received: June 17, 2002)

\begin{abstract}
In a recent Letter Bray and Blythe have shown that the survival probability $P_{A}(t)$ of an $A$ particle diffusing with a diffusion coefficient $D_{A}$ in a $1 \mathrm{D}$ system with diffusive traps $B$ is independent of $D_{A}$ in the asymptotic limit $t \rightarrow \infty$ and coincides with the survival probability of an immobile target in the presence of diffusive traps. Here we show that this remarkable behavior has a more general range of validity and holds for systems of an arbitrary dimension $d$, integer or fractal, provided that the traps are "compactly exploring" the space, i.e. the "fractal" dimension $d_{w}$ of traps' trajectories is greater than $d$. For the marginal case when $d_{w}=d$, as exemplified here by conventional diffusion in $2 \mathrm{D}$ systems, the decay form is determined up to a numerical factor in the characteristic decay time.

PACS No: 05.40.-a, 02.50Ey, 82.20.-w
\end{abstract}

Trapping $A+B \rightarrow B$ and recombination $A+B \rightarrow 0$ reactions (TR and RR) involving randomly moving $A$ and $B$ particles which react "when they meet" at a certain distance $b$ are ubiquitous in nature. A few stray examples include quenching of delocalized excitations, coagulation, recombination of radicals, charge carriers or defects, or biological processes related to population survival [1].

In recent years there has been much interest in the long-time behavior of these processes, following a remarkable discovery [2, 3, 4, 5, 6, 6, 8, 8, 9, 10] of many-particle effects, which induce essential departures from the conventionally expected behavior [1].

A pronounced deviation from the text-book predictions was found for the diffusion-controlled RR in case when initially the particles of the $A$ and $B$ species are all distributed at random with strictly equal mean densities $n_{0}$. It has been first shown [2] and subsequently proven [3, 1 that as $t \rightarrow \infty$ the mean density $n(t)$ follows $n(t) \sim \alpha_{d} n_{0}^{1 / 2}(D t)^{-d / 4}$, where $d$ is the space dimensionality, $\alpha_{d}$ is a constant and $D=D_{A}+D_{B}$ is the sum of particles' diffusion coefficients. This law contradicts the decay law obtained within the Smoluchowski approach $(\mathrm{SA}): n(t) \sim 1 / \phi_{b}^{(d)}(t)$ 四, where, as $t \rightarrow \infty$,

$$
\phi_{b}^{(d)}(t)=\int_{0}^{t} d \tau K_{S}(\tau) \sim\left\{\begin{array}{l}
4 \sqrt{D t / \pi}, \quad \mathrm{d}=1, \\
\frac{4 \pi D t}{\ln \left(4 D t / b^{2}\right)}, \mathrm{d}=2, \\
4 \pi D b t, \quad \mathrm{~d}=3,
\end{array}\right.
$$

$K_{S}(\tau)$ being the $d$-dimensional Smoluchowski-type "constant", defined as the flux of diffusive particles through the surface of an immobile sphere of radius $b$.

For the TR two situations were most thoroughly studied: the case when $A \mathrm{~s}$ diffuse while $B \mathrm{~s}$ are static, and the situation in which the $A$ s are immobile while $B$ s diffuse - the so-called target annihilation problem (TAP). In the case of static, randomly placed (with mean density $\rho$ ) traps the $A$ particle survival probability $P_{A}(t)$ shows a non-trivial, fluctuation-induced behavior [3, $4,5,6,6,0,8,9,10]$

$$
\ln P_{A}(t) \sim-\rho^{2 /(d+2)}\left(D_{A} t\right)^{d /(d+2)}, \quad t \rightarrow \infty,
$$

which is intimately related to many fundamenal problems of statistical physics [3, 1, $, 0,6,7,8,9,9,10,11$.

Survival probability $P_{\text {target }}(t)$ of an immobile target $A$ of radius $b$ in presence of point-like diffusive traps $B$ (TAP) can be calculated exactly for any $d$ (see Refs. 14 and [3, 12, 13]):

$$
P_{\text {target }}(t)=\exp \left(-\rho \phi_{b}^{(d)}(t)\right)
$$

where $\phi_{b}^{(d)}(t)$ obeys Eq.(1) with $D_{A}=0$. Decay forms in systems with hard-core interactions between $B \mathrm{~s}$ 15] or with fluctuating chemical activity [16] have been also derived.

On contrary, the physically most important case of TR when both $A$ s and $B$ s diffuse was not solved exactly. It has been proven [4] that here $P_{A}(t)$ obeys

$$
\ln P_{A}(t)=-\lambda_{d}\left(D_{A}, D_{B}\right) \times\left\{\begin{array}{l}
t^{1 / 2}, \quad \mathrm{~d}=1, \\
\frac{t}{\ln (t)}, \quad \mathrm{d}=2, \\
t, \quad \mathrm{~d}=3,
\end{array}\right.
$$

which equation defines its time-dependence exactly. On the other hand, the factor $\lambda_{d}\left(D_{A}, D_{B}\right)$ remained as yet an unknown function of the particles' diffusivities and $d$. Since the time-dependence of the function on the rhs of Eq.(4) follows precisely the behavior of $\int^{t} d \tau K_{S}(\tau)$, one might expect that the SA provides quite an accurate description for this situation and following its spirit to set $D_{A}=0$ supposing that traps diffuse with the diffusion coefficient $D=D_{B}+D_{A}$. As a matter of fact, it has been 
often tacitly assumed that when both of species diffuse $P_{A}(t)$ obeys Eq.(3) with $\phi_{b}^{(d)}(t)$ defined by Eq.(1) with $D=D_{A}+D_{B}$. On the other hand, it has been shown that $\lambda_{d}\left(D_{A}, D_{B}\right)$ is less than the corresponding prefactor in $K_{S}(t)[3]$ and that it may be bounded by a non-analytic function of $D_{A}$ and $D_{B}$ [17]. A perturbative approach for calculation of $\lambda_{d}\left(D_{A}, D_{B}\right)$, as well as corrections to the $\mathrm{SA}$ in 1D systems were presented [13]. It has been also noticed that $\lambda_{d}\left(D_{A}, D_{B}\right)$ is not a function of $D=D_{A}+$ $D_{B}$ only, since the diffusion-reaction equation are not separable 13. This lack of knowledge of the precise form of $\lambda_{d}\left(D_{A}, D_{B}\right)$, of course, constitutes an annoying gap in the general understanding of the fluctuation phenomena in chemical kinetics.

In a recent Letter Bray and Blythe (BB) 18 made a considerable step towards the solution of this general case by showing that, surprisingly, the survival probability of an $A$ particle diffusing in a 1D system with diffusive traps is independent of $D_{A}$ in the asymptotic limit $t \rightarrow \infty$ and coincides with the survival probability of an immobile target in the presence of diffusive traps, Eq.(3). The convergence to this asymptotic result might be, however, rather slow as shows the comparison [18] with extensive numerical simulations [19].

One may, however, pose quite a legitimate question whether such a remarkable result is constrained to physically not very realistic $1 \mathrm{D}$ systems with conventional diffusion or if it is just a particular case of a more general behavior which persists also for higher $d$ ?

In this Letter we show that indeed this remarkable result holds for a more general case. Namely, we show that for systems of an arbitrary dimension $d$, integer or fractal, the large- $t$ behavior of the survival probability of a randomly moving $A$ particle in the presence of randomly moving traps is given exactly by the solution of the exactly solvable TAP, provided that the traps are "compactly exploring" the space; in other words, the "fractal" dimension $d_{w}^{(B)}$ of traps' trajectories is greater than $d$ [20]. For lattice random walks, this corresponds to situations in which random walks are recurrent [21]. Random motion with $d_{w}^{(B)}>2$ is widespread in nature and is most often encountered in porous and disordered systems, amorphous and polymer materials [21], for which systems it will take place in two and even three dimensions (see, e.g., Refs. [20,22] and [23]). Finally, we examine the behavior in the marginal case when $d_{w}^{(B)}=d$, as exemplified here by conventional diffusion in 2D systems, and show that here the decay form can be determined up to a numerical factor in the characteristic decay time.

Consider a $d$-dimensional volume $V$ containing a single mobile $A$ particle of radius $b$ and $N$ point-like traps $B$. Let $\mathbf{X}_{t}$ be the vector denoting the $A$ particle position at time moment $t$, while $\mathbf{x}_{t}^{(j)}, j=1, \ldots, N$, be the corresponding vector denoting the position of the $j$-th trap. Introducing two auxiliary indicator functions

$$
\delta_{b}(\mathbf{x})=\left\{\begin{array}{ll}
1, & |\mathbf{x}| \leq b, \\
0, & \text { otherwise }
\end{array} \quad I(y)= \begin{cases}1, & y=0 \\
0, & \text { otherwise }\end{cases}\right.
$$

one writes $P_{A}(t)$ down formally as follows:

$$
P_{A}(t)=E\left\{\prod_{j=1}^{N}\left\langle I\left(\int_{0}^{t} \delta_{b}\left(\mathbf{X}_{\tau}-\mathbf{x}_{\tau}^{(j)}\right) d \tau\right)\right\rangle_{\left\{\mathbf{x}_{t}^{(j)}\right\}}\right\},
$$

where the symbol $E\{\ldots\}$ denotes averaging with respect to the A particle trajectories, while the brackets with the subscript $\left\{\mathbf{x}_{t}^{(j)}\right\}$ stand for averaging with respect to the trajectories of the the $j$-th trap. Note that Eq.(6) applies for any type of motion provided that the point-like traps are ignorant of each other and thus move independently. In the limit $N, V \rightarrow \infty(N / V=\rho)$ one has

$$
P_{A}(t)=E\left\{\exp \left(-\rho \int d \mathbf{x}_{0}\left\langle I^{\prime}\left(\mathbf{X}_{t}, \mathbf{x}_{t}\right)\right\rangle_{\left\{\mathbf{x}_{t}\right\}, \mathbf{x}_{t=0}=\mathbf{x}_{0}}\right)\right\},
$$

where brackets denote now averaging with respect to the trajectories of a single trap $B$ whose starting point is at position $\mathbf{x}_{0}$, while $I^{\prime}\left(\mathbf{X}_{t}, \mathbf{x}_{t}\right)$ is the indicator function

$$
I^{\prime}\left(\mathbf{X}_{t}, \mathbf{x}_{t}\right)=1-I\left(\int_{0}^{t} \delta_{b}\left(\mathbf{X}_{\tau}-\mathbf{x}_{\tau}\right) d \tau\right)
$$

which shows whether two given realizations of trajectories $\mathbf{X}_{t}$ and $\mathbf{x}_{t}$ have "intersected" each other at least once within the time interval $[0, t]$. Note that averaging over trajectories $\mathbf{x}_{t}$ in the exponential is to be taken for fixed $\mathbf{X}_{t}$, and after performing such an averaging we have to average an exponential of the result with respect to the trajectories $\mathbf{X}_{t}$, which represents a fairly complex mathematical problem. Such a complexity emphasises, of course, the significance of the BB result.

Now, BB have noticed [18], although not proven rigorously, that the $A$ particle will on average survive longer if it stays still than if it diffuses. They have also furnished some arguments in favor of this statement showing that this is true for systems with a finite number of traps since here the lowest value of the decay exponent corresponds to $D_{A}=0$. In other words, it means that $P_{A}(t)$ in Eq.(7) is bounded from above by

$$
P_{A}(t) \leq P_{\text {target }}(t)=\exp \left(-\rho \phi_{b}^{(d)}(t)\right)
$$

where $\phi_{b}^{(d)}(t)$ is given by

$$
\begin{aligned}
& \phi_{b}^{(d)}(t)=\int d \mathbf{x}_{0}\left\langle\left[1-I\left(\int_{0}^{t} \delta_{b}\left(\mathbf{x}_{\tau}\right) d \tau\right)\right]\right\rangle_{\left\{\mathbf{x}_{t}\right\}, \mathbf{x}_{t=0}=\mathbf{x}_{0}} \\
= & \int d \mathbf{x}_{0}\left\langle\left[1-I\left(\int_{0}^{t} \delta_{b}\left(\mathbf{x}_{\tau}-\mathbf{x}_{0}\right) d \tau\right)\right]\right\rangle_{\left\{\mathbf{x}_{t}\right\}, \mathbf{x}_{t=0}=0}
\end{aligned}
$$

Note also that Eq.(9) should hold for any type of random motion, not necessarily only for conventional diffusion. 
A lower bound on $P_{A}(t)$ can be constructed in the following way [18]: One notices first that all terms in the product in Eq.(6) are positive definite and hence, if one performs averaging of Eq.(6) not over all possible realizations of trajectories $\mathbf{X}_{t}$ and $\mathbf{x}_{t}^{(j)}$, but only over some restricted subset, one arrives at a lower bound on $P_{A}(t)$. Following Ref. [18], we define this subset as follows: let us suppose that initially the $A$ particle has been located at the origin while all traps were uniformly spread in a $d$-dimensional system such that the trap nearest to the origin appeared at distance $l$ from it. Then, we perform averaging only over such trajectories of the $A$ particles which do not leave within the time interval $[0, t]$ the volume of radius $l$ centered around the origin, and such trajectories of traps $B$ (which all initially are uniformly distributed outside of this volume) do not enter there within the time interval $[0, t]$. For such trajectories

$$
\prod_{j=1}^{N} I\left(\int_{0}^{t} \delta_{b}\left(\mathbf{X}_{\tau}-\mathbf{x}_{\tau}^{(j)}\right) d \tau\right) \equiv 1
$$

and hence, the following lower bound is valid

$$
\begin{aligned}
P_{A}(t) & \geq \exp \left(-V_{d} \rho l^{d}\right) \times \operatorname{Prob}\left(\max \left\{\left|\mathbf{X}_{\tau}\right|\right\}<l \mid \tau \in[0, t]\right) \\
& \times \operatorname{Prob}_{j}\left(\min \left\{\left|\mathbf{x}_{\tau}^{(j)}\right|\right\}>l \mid \tau \in[0, t]\right),
\end{aligned}
$$

where $V_{d}$ denotes the volume of a $d$-dimensional sphere of a unit radius, while two other multipliers stand for the probability that the $A$ particle does not leave a sphere of radius $l$ within the time interval $[0, t]$ and the probability that neither of traps, initially uniformly distributed with mean density $\rho$ outside this sphere, enters this sphere up to time $t$. Note that exactly the same lower bound has been already proposed in Refs. 224] and [17.

Now, let us suppose that the mean-square displacement of the $A$ particle obeys $<\mathbf{X}_{t}^{2}>\sim\left(D_{A} t\right)^{2 / d_{\omega}^{(A)}}$, while the MSD of traps follows $<\mathbf{x}_{t}^{2}>\sim\left(D_{B} t\right)^{2 / d_{\omega}^{(B)}}$, $d_{\omega}^{(A)}$ and $d_{\omega}^{(B)}$ being the "fractal" dimensions of the $A$ particle and traps trajectories, respectively. For conventional diffusion of has $d_{\omega}^{(A)}=d_{\omega}^{(B)} \equiv 2$ for any $d$. Under quite general conditions, for $D_{A} t \gg l_{\omega}^{d_{\omega}^{(A)}}$ the probability $\operatorname{Prob}\left(\max \left\{\left|\mathbf{X}_{\tau}\right|\right\}<l \mid \tau \in[0, t]\right)$ can be estimated as [21]

$$
\operatorname{Prob}\left(\max \left\{\left|\mathbf{X}_{\tau}\right|\right\}<l \mid \tau \in[0, t]\right) \sim \exp \left(-\beta_{d}\left(D_{A} t\right) / l^{d_{\omega}^{(A)}}\right),
$$

where $\beta_{d}$ is a constant dependent on the type of random motion and $d$. On the other hand, one readily notices that $\operatorname{Prob}_{j}\left(\min \left\{\left|\mathbf{x}_{\tau}^{(j)}\right|\right\}>l \mid \tau \in[0, t]\right)$ is just the probability that an immobile target of radius $l$ survives until time $t$ in the presence of randomly moving traps, i.e.

$$
\operatorname{Prob}_{j}\left(\min \left\{\left|\mathbf{x}_{\tau}^{(j)}\right|\right\}>l \mid \tau \in[0, t]\right)=\exp \left(-\rho \phi_{l}^{(d)}(t)\right),
$$

where $\phi_{l}^{(d)}(t)$ is defined by Eqs.(10) with $b$ replaced by $l$. We turn next to the most delicate point of our analysis. We note first that the definition in the first line in Eq. (10) allows to express $\phi_{l}^{(d)}(t)$, in virtue of the Gauss theorem, as a time-integral of $K_{S}(t)$ (see Ref. [3]). On the other hand, the definition in the second line in Eq.(10) shows that $\phi_{l}^{(d)}(t)$ can be thought of as the mean volume swept by randomly moving fictitious particle of radius $l$ during time $t$, i.e. the mean volume of the so-called "Wiener sausage" (see, e.g. Ref. [17). Its lattice counterpart is known as the mean number of distinct sites visited [21]. General properties of such a volume for different types of random motion have been first analysed in the pioneering papers by de Gennes [20], in which he studied $\mathrm{RR}$ involving polymerized particles and $\mathrm{TR}$ on the percolation cluster. As well, behavior of $\phi_{l}^{(d)}(t)$ have been discussed at length in Ref. [22] within the context of the polymer-free voids distribution in polymer solutions. It has been shown that depending on the relation between $d_{\omega}^{(B)}$ and $d$, two completely different types of behavior may be observed. The first type of behavior occurs when $d_{\omega}^{(B)}<d$. In this case $\phi_{l}^{(d)}(t)$, called by de Gennes as the "exploration volume", is smaller than the volume $\mathbf{x}_{t}^{d}$ where the particle is confined. This case is called the case of non-compact exploration and here $\phi_{l}^{(d)}(t) \sim \gamma_{d} t$, where the prefactor $\gamma_{d}$ is some function dependent on $d$ and the type of random motion. In this case $\gamma_{d}$ is proportional to some positive power of $l$ ! For lattice random walks this regime takes place when random walks are non-recurrent 21]. In the opposite case when $d_{\omega}^{(B)}>d$ the behavior is completely different. Here, the exploration volume $\phi_{l}^{(d)}(t)$ increases sublinearly with time, the trajectories are spatially more confined and most of space inside the volume $\mathbf{x}_{t}^{d}$ is indeed visited. This case is called the case of "compact exploration" (recurrent random walks for the lattice counterparts) and here

$$
\phi_{l}^{(d)}(t) \sim\left(\left|\mathbf{x}_{t}\right|\right)^{d} \sim\left(D_{B} t\right)^{d / d_{\omega}^{(B)}}, \quad t \rightarrow \infty
$$

What is most important in this case is that the prefactor in this asymptotic law is independent of $l$ ! Note also that such a behavior is compatible with the Alexander-Orbach result $\phi_{l}^{\left(d=d_{f}\right)}(t) \sim t^{d_{f} / d_{\omega}^{(B)}}$ for anomalous random walk on fractal lattices of dimension $d_{f}$ [25].

Now, we note that the function on the rhs of Eq.(12) is valid for any value of $l$ and consequently, the "best" lower bound would correspond to such $l$ which provides its maximal value. Focussing next solely on the case of random motion with compact exploration, we note that in this case the leading large- $t$ behavior of $\phi_{l}^{(d)}(t)$ is independent of $l$, and hence, we have to maximize only the product of the first two terms. This yields

$$
P_{A}(t) \geq \exp \left(-\alpha_{d}^{\prime} \rho^{1-z}\left(D_{A} t\right)^{z}\right) \exp \left(-\rho \phi_{l}^{(d)}(t)\right)
$$


where $\alpha_{d}^{\prime}$ is a constant, $z=d /\left(d+d_{\omega}^{(A)}\right)$ and the asymptotic behavior of $\phi_{l}^{(d)}(t)$ is defined in Eq.(15).

For compact exploration we have that $d<d_{\omega}^{(B)}$. On the other hand, on comparing the growth rate in the exponent in the first term on the rhs of Eq.(16) against the growth rate of $\phi_{l}^{(d)}(t)$ defined in Eq.(15), we infer that the second multiplier determines the overall decay in case when $d, d_{\omega}^{(A)}$ and $d_{\omega}^{(B)}$ obey: $d<d_{\omega}^{(B)}<d+d_{\omega}^{(A)}$, which reduces to simple condition of compact exploration for $d_{\omega}^{(A)}=d_{\omega}^{(B)}$. Further on, for $d, d_{\omega}^{(A)}$ and $d_{\omega}^{(B)}$ which obey the double side inequality, we have evidently that the leading terms in Eqs.(9) and (16) coincide, since $\phi_{b}^{(d)}(t)$ is asymptotically independent of $b$ and $\phi_{l}^{(d)}(t)$ does not depend on $l$. We infer thus that in this quite general case exact asymptotic solution for trapping reactions $A+B \rightarrow B$ in which both species move randomly is given by the solution of the corresponding immobile target annihilation problem, which represents a substantial generalization of the $\mathrm{BB}$ result 18 .

Finally, we analyze the behavior in the marginal case $d=d_{\omega}^{(B)}=d_{\omega}^{(A)}$ using as an example conventional diffusion in $2 \mathrm{D}$ systems. In this case, an asymptotical behavior of $\phi_{l}^{(d=2)}(t)$ is well-known [26]:

$$
\begin{aligned}
\phi_{l}^{(2)}(t) & =\frac{4 \pi D_{B} t}{\left(\ln \left(4 D_{B} t / l^{2}\right)-2 \gamma\right)}\left[1+\frac{A_{1}}{\left(\ln \left(4 D_{B} t / l^{2}\right)-2 \gamma\right)}\right. \\
& \left.+\mathcal{O}\left(\left(\ln \left(4 D_{B} t / l^{2}\right)-2 \gamma\right)^{-2}\right)\right]
\end{aligned}
$$

where $A_{1} \approx 0.423$ and $\gamma$ is the Euler constant. On the other hand, for standard diffusive motion one has that $\operatorname{Prob}\left(\max \left\{\left|\mathbf{X}_{\tau}\right|\right\}<l \mid \tau \in[0, t]\right)$ obeys Eq.(13) in which one sets $d_{\omega}^{(A)}=2$ and $\beta_{2}$ is the square of the first zero of the Bessel function $J_{0}(x)$. Now, since $\phi_{l}^{(2)}(t)$ is only weakly (logarithmically) dependent on $l$, one may assume that the value of $l$ which maximizes the lower bound for $D_{A} t \gg l^{2}$ is still determined by the derivative of the first two terms on the rhs in Eq. 12, , i.e. $l \sim\left(\beta_{2} D_{A} t / V_{2} \rho\right)^{1 / 4}$. Such an estimate shows then that in $2 \mathrm{D}$ systems with conventional diffusion the $A$ particle survival probability is bounded by:

$$
\begin{aligned}
1 & +\frac{A_{1}}{\ln \left(4 D_{B} t / b^{2}\right)}+\mathcal{O}\left(\ln ^{-2}(t)\right) \leq \\
& \leq \ln \left(1 / P_{A}(t)\right) \frac{\ln \left(4 D_{B} t / b^{2}\right)}{4 \pi D_{B} t \rho} \leq 2- \\
& -2 \frac{\left(\ln \left(4 / \beta_{2}\right)+\ln \left(V_{2} \rho b^{2}\right)+\ln \left(D_{B} / D_{A}\right)\right)}{\ln \left(4 D_{B} t / b^{2}\right)}+\mathcal{O}\left(\ln ^{-2}(t)\right)
\end{aligned}
$$

Hence, in this marginal case the suitably extended upper and lower bounds determine the decay form up to a numerical factor in the characteristic decay time.

The authors acknowledge helpful discussions with Professors Bray and Blythe on the matters of this paper.
[1] S.A.Rice, in Diffusion-Limited Reactions, edited by C.H.Bamford, C.F.H.Tipper and R.G.Compton, Comprehensive Chemical Kinetics Vol. 25 (Elsevier, Amsterdam, 1985)

[2] A.A.Ovchinnikov and Ya.B.Zeldovich, Chem. Phys. 28, 214 (1978); S.F.Burlatsky, Teor. Exp. Chem. 14, 343 (1978); D.Toussaint and F.Wilczek, J. Chem. Phys. 78, 2642 (1983); S.Redner and K.Kang, Phys. Rev. Lett. 51, 1729 (1983); G.Zumofen, A.Blumen and J.Klafter, J. Chem. Phys. 82, 3198 (1985)

[3] S.F.Burlatsky and A.A.Ovchinnikov, Zh. Eksp. Teor. Fiz. 92, 1618 (1987) [Sov. Phys. JETP 65, 908 (1987)]

[4] M.Bramson and J.L.Lebowitz, Phys. Rev. Lett. 61, 2397 (1988); J. Stat. Phys. 62, 297 (1991)

[5] B.Ya.Balagurov and V.T.Vaks, Zh. Eksp. Teor. Fiz. 65, 1939 (1973) [Sov. Phys. JETP 38, 968 (1974)]

[6] M.D.Donsker and S.R.S.Varadhan, Commun. Pure Appl. Math. 28, 525 (1975)

[7] L.A. Pastur, Theor. Math. Phys. 32, 88 (1977)

[8] P. Grassberger and I. Procaccia, J. Chem. Phys. 77, 6281 (1982)

[9] R.F. Kayser and J.B. Hubbard, Phys. Rev. Lett. 51, 6281 (1982)

[10] T.C. Lubensky, Phys. Rev. A 30, 2657 (1984); S.R. Renn, Nucl. Phys. B 275, 273 (1986); Th.M. Nieuwenhuizen, Phys. Rev. Lett. 62, 357 (1989)

11] C. Monthus, G. Oshanin, A. Comtet and S.F. Burlatsky, Phys. Rev. E 54, 231 (1996)

[12] A.Blumen, G.Zumofen and J.Klafter, Phys. Rev. B 30, 5379 (1984)

[13] A.Szabo, R.Zwanzig and N.Agmon, Phys. Rev. Lett. 61, 2496 (1988)

[14] M.Tachiya, Radiat. Phys. Chem. 21, 167 (1983)

[15] S.F.Burlatsky, M.Moreau, G.Oshanin and A.Blumen, Phys. Rev. Lett. 75, 585 (1995); D.P.Bhatia, M.A.Prasad and D.Arora, Phys. Rev. Lett. 75, 586 (1995)

[16] O.Bénichou, M.Moreau and G.Oshanin, Phys. Rev. E 61, 3388 (2000)

[17] A.M.Berezhkovskii, Yu.A.Makhnovskii and R.A.Suris, J. Phys. A 22, L615 (1989)

[18] A.J.Bray and R.A.Blythe, Exact Asymptotics for OneDimensional Diffusion with Mobile Traps, Phys. Rev. Lett., to appear; cond-mat/0205381

[19] V.Mehra and P.Grassberger, Phys. Rev. E 65, 050101(R) (2002)

[20] P.G. de Gennes, C. R. Acad. Sc. Paris 296 Série II, 881 (1983); J. Chem. Phys. 76, 3316 (1982)

[21] B.Hughes, Random Walks and Random Environments, (Oxford Science Publ., Oxford, 1995)

[22] G.Oshanin, S.F.Burlatsky and M.Moreau, Adv. Colloid and Interface Sci. 49, 1 (1994)

[23] G.Oshanin, S.Nechaev, A.M.Cazabat and M.Moreau, Phys. Rev. E 58, 6134 (1998); S.Nechaev, G.Oshanin and A.Blumen, J. Stat. Phys. 98, 281 (2000)

[24] S.Redner and K.Kang, J. Phys. A 17, L451 (1984) 
[25] S.Alexander and R.Orbach, J. Phys. (Paris) Lett. 43, L625 (1982)

[26] A.M.Berezhkovskii, Yu.A.Makhnovkii and R.A.Suris, J. Stat. Phys. 57, 333 (1989) 